\title{
Morphologic Complete Remission with Incomplete Blood Count Recovery
}

National Cancer Institute

\section{Source}

National Cancer Institute. Morphologic Complete Remission with Incomplete Blood

Count Recovery. NCI Thesaurus. Code C123596.

The disappearance of all cells with morphologic characteristics of cancer, accompanied by incomplete bone marrow recovery, as evidenced by persistent anemia, thrombocytopenia or neutropenia. 